\title{
Accent in Digital Humanities and Language Studies: The Case in Hong Kong
}

\author{
CHARLES LAM \\ Department of English, \\ The Hang Seng University of Hong Kong \\ charleslam@hsu.edu.hk \\ CATHERINE WONG \\ Department of English, \\ The Hang Seng University of Hong Kong
}

\begin{abstract}
This paper argues for the importance of the awareness of "DH accent" and demonstrates with examples in English studies how a localised variation of the curriculum facilitates students' learning in the classroom and at the curriculum level. This study identifies the problem that studies in digital humanities have focused on the AngloAmerican world. We demonstrate with an example in the Hong Kong context that even a curriculum of English language studies requires adaptation for the local needs, such as focus on second language learning and knowledge of contrastive grammar with the local language. To achieve these goals, instructors integrate materials that are tailored for students of language studies, who are typically proficient in humanistic argumentation and concepts but less fluent in digital skills. Use cases in teaching and examples of student projects are shown to illustrate the outcome of learning. The study presents important educational implication and direction for future research and education of the digital humanities.
\end{abstract}

Keywords: digital humanities; English; accent; pedagogy; Hong Kong

\section{INTRODUCTION}

The emergence of DH provides a platform for cross-disciplinary collaboration. It offers computer scientists an inspiration of a new direction of research questions, data scientists a new area of acquisition of text data and humanists a formal method of research. The intersection of these traditionally varied disciplines, however, has also created a diverse understanding of the level of involvement of the digital/technological support. On one hand, the digitalisation of humanities projects offers a scientific method to traditionally impressionistic analysis of humanistic contents (Risam \& Koh, 2013); on the other hand, the difference in trainings of various disciplines gives rise to a variety of approaches to seemingly similar problems. This paper discusses the case of implementation of DH in an English undergraduate programme in Hong Kong. In particular, we argue that greater awareness to integrate digitalisation in humanities and language studies is crucial for both students and instructors in contexts where English is not the native language for all students.

The philosophy behind our pedagogical practices is closely related to the notion of "DH accent", a term coined by Risam (2015), which aims to highlight the diversity across different regions. Accent is defined as "a variety of speech differing phonetically from other varieties" (Matthews, 1997). However, accent also often carries a connotation of mispronunciation and errors. In the context of teaching digital humanities, the problem is that such a negative connotation is counterproductive. The needs of students and researchers in different parts of the world are very different, this study therefore calls for attention to the accents of DH, so as to accommodate the needs of different regions. Although the different $\mathrm{DH}$ accents stem from linguistic differences (English vs. local languages in different regions in Asia), the significance 
is more far-reaching than choosing a different language as the medium of instruction. In the community of World Englishes (Kachru, 1992), different variations of English are maintained to be the result of adaptation to local needs and environments. Therefore, they are of equal value, and no variations should be treated as inferior to others. Similarly, researchers and instructors of digital humanities face very different research questions and challenges in different contexts. Therefore, the accents of DH in different regions are necessarily different. Specifically, we show our implementation in the English curriculum in higher education in Hong Kong. As a working definition, "DH accent" refers to the variation of research and instructional focus in digital humanities, both in terms of contents and methodology. This formulation takes cue from Risam (2017)'s concept of DH accent that aims to create "an approach that foregrounds the affordances rather than the limitations of its heterogeneity" (Risam 2017, p. 4). Similar notions have been discussed also in Earhart (2018) as "localized expression" in digital humanities. The case of university-level English curriculum is interesting because of its complex background (where students are expected to master a second language at near-native level), as well as its ubiquity across various regions in the world.

The mandatory bilingual education system in Hong Kong has trained its students to be experienced L2 speakers in English by the time they enter into university - these students often have had 15+ years of EFL/ESL training since kindergarten. However, the L2 classroom in their primary and secondary school education has also created in them an impression of English studies restricted to only that of second language acquisition - these students, even when choosing English as their major, have little expectation to the curriculum at the college level they are not necessarily interested in English literature and have definitely no prior knowledge of linguistics. With this unique phenomenon prevailing in the discipline within the local context, this paper proposes a method of blending technology into the curriculum and its goal is to identify a DH accent which pitches for the undergraduate level English curriculum in Hong Kong.

\section{DH ACCENT IN THE GLOBAL CONTEXT}

This section extends previous research and argues that accent in DH should be defined not narrowly in the operating language, but also by several other parameters such as the subject matter and its socio-cultural contexts. To derive such a definition, a theoretical framework is essential in ensuring a significant level of consistency of the understanding and application of DH across disciplines and contexts. We adopt Risam (2017)'s concept of "DH accent" as the theoretical framework of this paper. This is adopted for four principles: as in accent denoting language variation, 1) there is a variety of methods and subject matters in digital humanities, in both teaching and research; while accent usually carries a negative connotation of mispronunciation, 2) this range of variations in DH tends to be subject to criticism that the field "lacks a theoretical model for reflecting critically on its own instruments" (Fiormonte, 2012: 59). However, we argue that albeit the general belief of the "essence" of $\mathrm{DH}, 3$ ) being "a discipline and an academic discourse dominated materially by an Anglo-American élite and intellectually by a mono-cultural view" (Fiormonte, 2012, p. 59), its variations should be valued as the accent 4) which are "localised" and possessing property specific to context and subject matter that meets the local needs and environment. By surveying through previous research, we aim to explore the various possibilities of DH applications in the global context in order to recommend a DH accent which best serves the local needs of the undergraduate level English curriculum in Hong Kong.

The issue on geographic and linguistic diversity has been extensively discussed in Galina (2014). Galina provides a general overview of the state of the art and highlights that the 
DH community is internally diverse with researchers from various locations and language backgrounds. The significance of this diversity lies in the challenge that comes with it: The geographical diversity means that research projects address very different issues and audiences. Chen \& Hsueh (2014) discuss the regional development of DH in Taiwan, providing the distribution of 1,104 publications in DH. While their data show that many of the publications relate to social sciences, humanities and technology, they also indicate that some of these studies (196 out of 1,104) have referenced materials on Chinese and Japanese history (Chen \& Hsueh 2014, p. 22, table 6). This indicates that some DH projects are bound to be specific to their geographical locations. What it means for the global DH community is that the subject matter (e.g. East Asian history) is tightly intertwined with the origin of the research and research groups. In this globalized context, Earhart (2018) addresses how the dominance of the major centres of DH may lead to homogenisation and suggest that researchers should no longer "encapsulate all practices of digital humanities within a big tent or a centralized structure", and instead expect a more decentralised community. We further argue that such decentralisation is necessarily and technologically feasible. With the development of modern technology, most notably the development of cloud-based databases and language corpora, researchers are no longer bound by their physical location when conducting research. Libraries and databases being accessible online has been a standard practice for some years. Individual researchers may also sign up for web hosting services for free. This technological change and the convenience it brings also gives rise to opportunities for comparison across areas. Risam (2016), for example, has compared the development of black feminism in the contexts of Africa and North America. On the one hand, this type of comparative research would be more difficult to conduct without the globalised research community backed by technology. On the other hand, the study shows an example of how a decentralised and distributed community allows for a wider range in topics and themes in humanities. Towards the end of the article, Risam calls for "theorisation of the relationship between local and global in scholarship and practice". In this article, we aim at addressing the issue from a technological and pedagogical aspect, i.e. how the more integrated use of technology can foster a globalised research community, in addition to the apparent benefits of free information flow.

The other issue is the linguistic diversity that comes with the globalised DH community. Given the diversity in geographical location, the diversity in linguistic background is a natural consequence. In natural sciences, the common language of mathematics and quantified data is by and large universal. Learning the mathematical language and the skills in using quantified data are integral parts of learning the physical science. While English proficiency is important in many occasions, such as publishing in general and teaching at English-dominant environments, the language proficiency of the researcher often remains a tool of communication. Disciplines in the humanities, on the other hand, do not follow a uniform set of rules. Rather, the data and the arguments are often intertwined with the language used. In some cases, English is not merely a means of communication, but also the subject of discussion. This is most obvious in the cases of literary studies and linguistics. For a literary scholar, the subject of study, especially in the postcolonial context, can be a novel written in the English language by any Anglophone writer who uses a particular variant/dialect of English. For a linguist, it is common to discuss subtle differences between languages and dialects. In both cases, the issue of linguistic diversity is manifested not only in the language background of the authors, but also in the variation in the subject of study. What this distinction means for DH and accents in DH is that the language itself may directly affect the contents through the authors' assumptions of their audience. For instance, studies on the Chinese language are typically disseminated in conferences and journals in China or Taiwan. When similar projects on the Chinese language are presented in English, it is often the case that the authors have made the conscious decision to communicate with non-Chinese speakers about the language, possibly 
because of certain cross-linguistic value or generalisation on some common phenomena. While it is important to acknowledge the role of a lingua franca in the global context, the vast diversity in language may also be a barrier for specific topics. In many cases, the findings are only available in English outlets and therefore difficult to reach by readers that are not in the Anglophone centres, if not for the technical issues of paywall and resources of libraries. However, a tougher problem exists when the research findings are published in a more locally focused outlet and a language other than English. Consequently, the majority of English audience would not be aware of such findings. If the linguistic diversity was merely a fact from the diverse backgrounds of the authors, the barrier would be relatively easy to remove, simply through the use of or translation to English and other common lingua franca in academia. However, if the results are disseminated in a less common language, the knowledge might not reach its intended audience at all. The natural result of such a reality is that researchers would simply avoid using the regional/local languages and work primarily, if not entirely, in English. In turn, the research community becomes homogenised as most or all members decide to use English as the working language. It is therefore important to acknowledge and recognise the variations and accents in order to preserve the diversity.

Previous studies have recognised the dominance of the English language and called for more linguistic, geographic and cultural diversification (Galina, 2014; Mahony, 2018), which can bring diversity and, more importantly, inclusiveness to the DH community. In this context, Risam (2017) proposes a concept of "DH accent" that captures the diversity and specific features of DH across different regions without losing the universal perspective in DH. Similar to different regional accents and variations in English, which can be defined in several dimensions, such as their phonetic articulations, word choices and syntactic constructions, DH projects may also vary according to their regional needs. Risam cites her own example of the entry in Urdu Cyber Library and elucidates that this project brings different merits for different communities in different contexts. On one hand, the digitisation facilitates accessibility and new addition of "novel" contents for the global community. On the other hand, the project shows evidence to convince the local communities in India and Pakistan to support digitisation (Risam, 2017: 6). We consider this situation similar to accents in speech. In different contexts, the difference in phonetic realisations carries different undertones and connotations. An accent may represent a high-prestige variation in one context but a low-prestige in another. In some cases, an accent is merely an accent and not associated with any particular connotation or identity. We agree with Risam that DH studies are always accented. Building on this, we also maintain that the domination of the English-speaking variations in DH is also an accent, albeit a dominant one. What it means is that the non-dominant accents are equally valuable and may achieve different goals when put in different contexts. If it is the case that variations and different accents and variations in speech should be respected as equal in value, then it would be logical that $\mathrm{DH}$ accents are also equal in their status.

Given the overview of previous studies above, we argue that DH accents defined narrowly in language as either subject of investigation or medium of dissemination are inadequate. The present study aims to show that a broader notion of DH accent helps reconcile the differences and the underlying common thread in various disciplines of humanities.

\section{DH ACCENT IN THE CONTEXT OF ENGLISH LANGUAGE STUDIES IN ASIA}

The previous section advocates for the notion of $\mathrm{DH}$ accents to better accommodate and include the variety within the research community. Given that students and instructors are often nonnative speakers of English in this context, we detail the challenges and our efforts in tailoring the curriculum in order to suit the language proficiency and needs of the students. Although 
the discussion below is based on our experience of one institution in Hong Kong, we believe that many aspects can be generalised to other areas in Asia.

We describe a common challenge faced by Asian students and scholars as a "double duty problem" in using and studying English at the same time. In Hong Kong, over $90 \%$ of the population is ethnically Chinese and speaks Cantonese or other Chinese dialects as their first language (HKSAR Census 2017). Because of the colonial history, children typically begin learning English as a second language from kindergarten, and English remains a compulsory subject in the school system. As a result, students have typically received over a decade of English language training by the time they enter the university or other post-secondary institutions. They are often expected to be proficient, even though they are not native speakers of English. For students who choose to study English or language-related humanities subject at the university level, the expectation of their English proficiency is even higher. A great challenge is that, as non-native speakers of English, students' language and cultural proficiencies are not directly comparable with native speakers. For scientific or business disciplines, the difference in language proficiency may not be significant. However, as linguistic or literary studies often require nuanced understanding of texts and socio-historical backgrounds, the challenge for non-native speakers is more pronounced. This "double duty" challenge is manifested in, for example, semantics and pragmatics in linguistics, which often require understanding of ambiguity or context sensitivity of sentences. Since textbooks and research articles are mostly in English, students in Hong Kong have to achieve a high level of their second language, in order to understand the examples and the linguistic theories. In a similar manner, students will find themselves in need of bridging knowledge of the history of English, when they study English literary texts in the 17th and 18th centuries or awareness of the cultural literacy and differences, when they study Anglophone writings emanated from different parts of the world (e.g. traditional British and American literature vs the English language literature from the Caribbean). Oftentimes this problem is accepted as part of the teaching and learning process without much discussion. However, implicitly accepting the double duty as part of the learning can be counterproductive for students' learning, because the mixed nature of the problem can often be a source of confusion and frustration for students. We argue that it is important to acknowledge the difficulty faced by students for practical reasons. On one hand, this helps students understand the expectation from the instructors and courses. For instance, first year students may falsely assume their English courses to contain more grammar drills or practical writing, hence missing the training in close reading and interpretation. On the other hand, for students to understand that they are attempting two tasks at the same time would also help them understand the nature of their difficulties. For students who are weak in English proficiency, instructors can respond directly to the problems in the lower level linguistic problems. For students who are proficient in English but weak in understanding novel concepts, instructors can then focus on the contents, rather than the issues with second language learning. In most cases, students may not readily identify the type of their difficulties, and this can often be the reason for frustration and lack of interest. For this reason, we argue that the double duty problem is often assumed, but not adequately recognised or explicitly addressed.

The double duty of acquiring the language as a means of communication and discussing the language as a subject of investigation leads instructors of humanities in Asia to adjust their curriculum to suit students' needs. More specifically, instructors would need to make sure at every step that students can understand the materials and discuss the issues at hand, without any language barrier. This is particularly difficult when there is pressure to complete the planned materials. We believe this double duty problem applies to all instructors in the typical Asian context where English is not the students' first language, regardless of whether they take a digital or traditional approach. What we argue in this paper is that scholars who adopt a digital 
approach may find it easier to advocate for the importance of English studies and humanities where only the instrumental use of English (such as basic communication purposes for conducting business) is emphasised for employment prospects. Digital approaches to humanities often require students to combine textbook knowledge to data or situations that are more closely related to their own lives. For example, students in corpus linguistics or computational linguistics are often required to apply their skills to novel data. Examples and exercises from textbooks are often based on English data, since the more popular introductory texts are written for English-speaking audiences. When students with non-Anglophone backgrounds are asked to apply their knowledge to new data that are related to their own lives, it is common for them to take apply methods in corpus linguistics to data in their own languages, or English writing data from non-native users like themselves. These examples show how digital approaches or incorporation of information technology can promote application of knowledge and, in turn, extending the theoretical knowledge to languages or speakers that are typically not discussed in standard texts. In other words, the use of digital tools naturally encourages students to actively apply their knowledge to situations that bear more local relevance. To facilitate this objective, the notion of $\mathrm{DH}$ accent is essential in guiding instructors and scholars in their curriculum. In fact, there is an upward trend of integrating DH in English studies in Asian EFL context and studies present a promising picture that localised DH applications benefit Asian students in their English studies (Hamat \& Hassan, 2019; Lin \& Lin, 2019). To further illustrate our claim, $m$ ore examples from our own experience will be provided in section 4.

Like other humanities disciplines, an important objective of English studies is the general analytical skills that provide students with critical views to the world. Scholars have long recognised the pedagogical value of analytical and critical thinking that humanities disciplines can bring to students. In the context of $\mathrm{DH}$, it has also been argued that critical thinking and evidence-based argumentation because of the incorporation of digital components to humanistic inquiries (Schreibman, Siemens \& Unsworth 2008; Gold 2012). In the more traditional humanities, students are exposed to philosophical and abstract concepts that might not be immediately tangible. In semantics and pragmatics, students typically learn about the cooperative principle by Grice (1957) that aims to theorise how pragmatic meanings may be perceived, even though the intention is not explicitly stated. A textbook example would describe a scenario, where someone wants the salt from across the table and politely asks "Can you pass the salt?". While the utterance is a yes-no question, hearers are often able to understand the intention of the speaker and react accordingly. Students are then expected to be able to identify similar scenarios, where intended meanings are conveyed without being explicitly stated. Alternatively, they may also be asked to analyse other scenarios and argue to support their analysis. Given that pragmatic meanings can sometimes be partially dependent on the cultural background, instructors would adjust the examples as well as explanations to suit students' background and expectation. In the Asian context, this often means including reference to popular cultures in the region, or other social and political events that are of local relevance. Although the practice often results in more materials for students, the additional illustration also ensures deeper understanding of the theoretical concepts. Furthermore, examples that are more closely related to students' everyday life can be useful for topics of assignments and projects, which effectively assess students' ability to apply their knowledge to analyse novel examples. In other words, there are challenges in introducing novel concepts to students with little or no background of Anglophone cultures, instructors may adapt examples closer to the local culture and in turn deepen students' understanding of the concepts. In the context of DH pedagogy, this practice can often translate to assignments that require students to collect data and examples that illustrate the theoretical concepts. Depending on the nature of individual courses or assignments, the technological requirements may vary. In 
section 4, we will demonstrate some examples of assignments and submissions from our students in the past.

In addition to the pedagogical values of humanities, English language education in Asia also means empowering students to connect and communicate to other parts of the world. Given the linguistic diversity within Asia and no Asian language is as dominant and widely spoken as English, the mastery of English and the ability of discuss more abstract issues in cultures and diversity is important for intercultural communication. Since English language scholars from Asia are often not native speakers of English, one may question the usefulness in "competing" with native speakers in the discipline where Asian users face a natural disadvantage. For students, it is also reasonable to ask why one should study English language in Asia. Our answer to this comes directly from DH accent: Although students may not have native level proficiency in English, they do have the advantage of possessing deep understanding of the more globalised Anglophone cultures and their own local cultures. Mahony (2018) advocates for cultural diversity, largely based on multilingualism. The case of Hong Kong, where most students are trilingual (Cantonese, English and Mandarin), shows that proficiency in multiple languages paths the way to obtain information and learn from a wider range of resources. This strongly supports multilingualism for its benefits in both cultural diversity and economic prosperity. The multilingualism in Hong Kong also suggests that including more languages is only a first step. In the higher education setting in Hong Kong, where multilingualism is an everyday fact, students tend to demonstrate an internalised division of labour between Cantonese, English and Mandarin. Oftentimes, these languages are assigned a specific domain and students are reluctant to break the division, although it is clear that students are in general competent in both languages and can communicate with them casually as well as professionally. For example, English is often the default language choice in email communications among professionals or students in formal communications, and Cantonese in more casual or oral communications. In a broader sense, English language studies enable students and scholars to communicate and negotiate between communities of different cultures.

\section{BLENDING DIGITAL HUMANITIES AND LANGUAGE STUDIES}

To illustrate how we implement accented DH in our classes, this section discusses two use cases, where humanistic inquiry is blended with technology. Both cases highlight the incorporation of digital methods in questions that are traditionally answered by close reading of textual data. These cases also show how such an approach enhances instruction and scholarship. With our examples, we also demonstrate that the use of digital methods is key to promoting accented DH through incorporating novel data, which are not usually included in standard textbooks.

Linguistic typology is the study of the features of different languages in the world. In the Hong Kong context, typology is traditionally under the label of "contrastive analysis" as part of English studies. In the past, this has often been treated as a course on how to analyse grammatical errors in English made by native speakers of Chinese languages, and bears a connotation of teaching what is considered standard English to non-native speakers. Since the notion of World Englishes (Kachru 1992) becomes more popular among linguists, different variations and accents of English are considered different but equally valid "Englishes". Kachru's conceptualisation of English in the three circles (inner, outer and expanding) enables scholars and students to acknowledge and value different variations, not only for ideological reason, but more importantly for pragmatic reasons. In the case of Hong Kong, students use English to communicate with speakers from the inner circle of English (e.g. United Kingdom or United States), as well as speakers from outer and expanding circles, which includes those 
from Hong Kong. However, in their pre-college English education, students are often trained to accept only one of the standards (British English and Received Pronunciation in the case of Hong Kong). The adaptation of data-driven and digital methods is important in convincing students that variation is part of language use in the real world. In the traditional setting, the data are provided in text-based tables, and the course focuses on how the first language of students may cause their error in English. In order to illustrate the pluricentric view of "World Englishes" that variants of English are equally valid, corpus data have been used to show variations in word choice, grammar and pronunciation. For example, students are asked to compare various English corpora, and discuss differences across English variations. In the Hong Kong context, it is commonly believed that British spelling is more often used, because of the colonial history. However, a quick google search would reveal that, for instance, the American -ize spelling (as in "analyze" or "monetize") is more frequent in websites with the Hong Kong based ".hk" domains. This is a perfect example of how DH enables students to debunk a common misconception with empirical data. Similar to the use of English corpora to demonstrate English variations, the World Atlas of Language Structures (WALS, https://wals.info) in a language typology class helps students understand that language may contrast with each other in many different parameters. WALS includes 192 features in aspects such as phonology, morphology, syntax and lexicon. Users can visualise the distribution of the linguistic feature through maps. Fig. 1 below shows the map of basic word orders, which is useful in illustrating the general distribution.

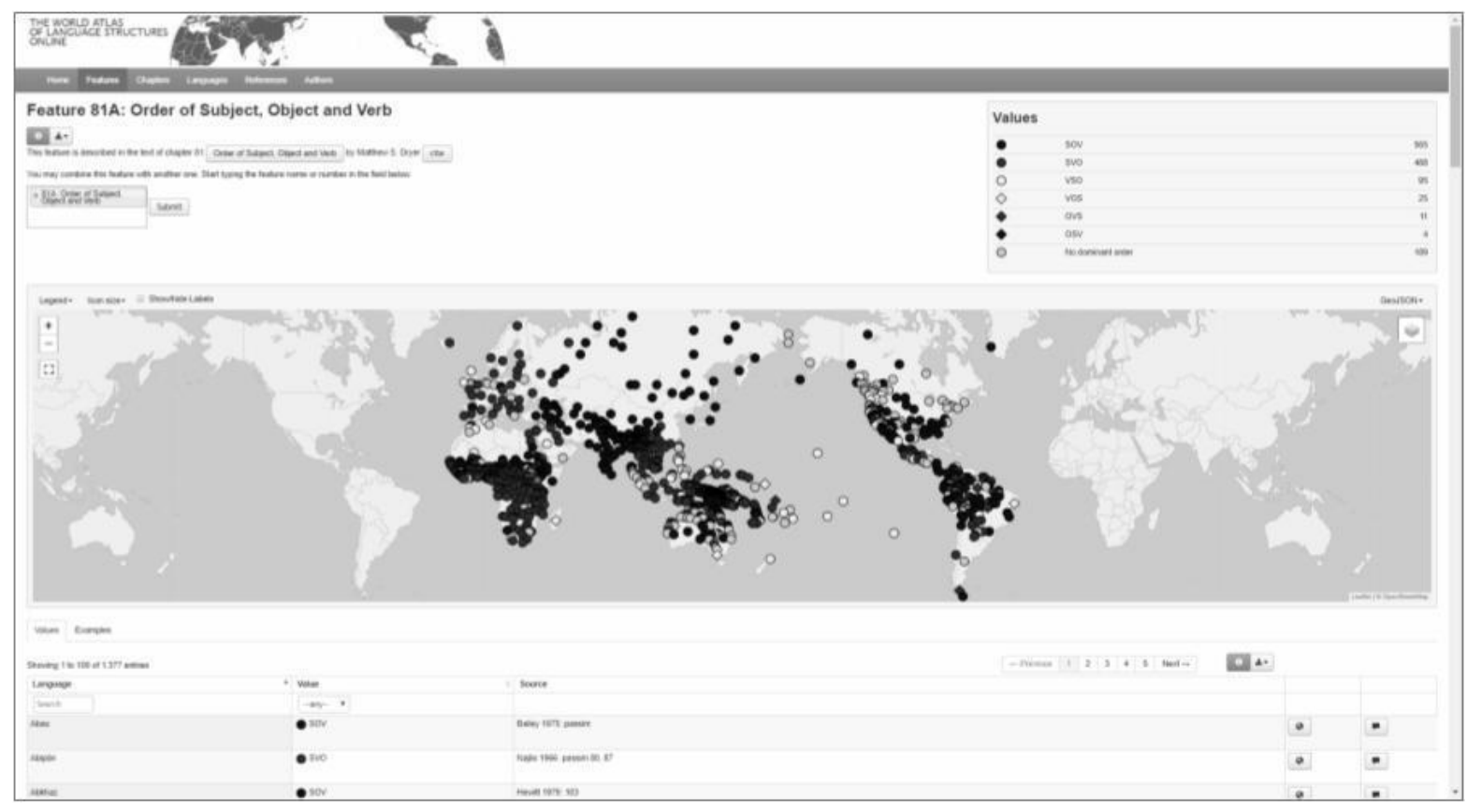

FIGURE 1. Word order distribution (http://wals.info, accessed on Dec 11, 2018)

Without the visualisation tool of linguistic features, instructors often have to manually create tables to illustrate the results, which is time-consuming and more difficult for students to understand. This case highlights how DH, especially when combined with a localised need to suit students' interests and background, can enhance learning in both breadth and depth. Researchers can also investigate a wider range of data efficiently and better understand the distribution of the linguistic features.

Incorporating empirical data and visualising them can help students see the larger picture. This, in turn, helps them become unbiased of their own experience. For example, it is a common believed that tonal languages (i.e. pitch difference in speech is used to distinguish 
lexical, word-level meaning, such as Cantonese) are rare. This is likely to be a confirmation bias that comes from the fact that popular foreign languages (e.g. English, Spanish, French, Japanese and Korean) are often non-tonal. Fig. 2 below from WALS shows that tonal languages make up over $40 \%$ of the investigated languages and they are far from the minority.

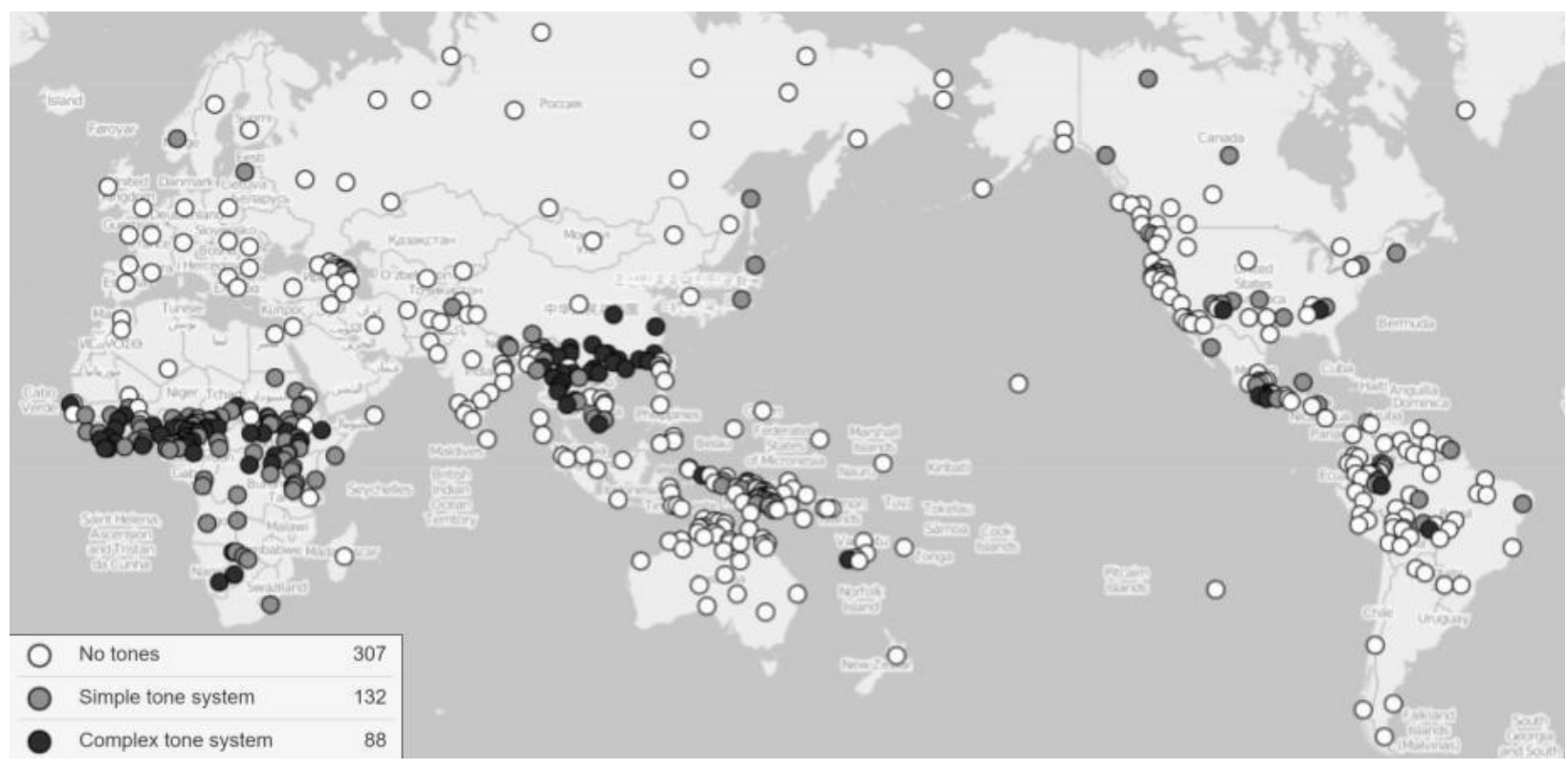

FIGURE 2. Distribution of Tonal Languages (Feature 13A in WALS, accessed on Dec 11, 2018)

Without the visualisation tool of linguistic features, instructors often have to manually create tables to illustrate the results, which is time-consuming and more difficult for students to understand. This case highlights how $\mathrm{DH}$, especially when combined with a localised need to suit students' interests and background, can enhance learning in both breadth and depth. Researchers can also investigate a wider range of data efficiently and better understand the distribution of the linguistic features.

Incorporating empirical data and visualising them can help students see the larger picture. This, in turn, helps them become unbiased of their own experience. For example, it is a common belief that tonal languages (i.e. pitch difference in speech is used to distinguish lexical, word-level meaning, such as Cantonese) are rare. This is likely to be a confirmation bias that comes from the fact that popular foreign languages (e.g. English, Spanish, French, Japanese and Korean) are often non-tonal. Fig. 2 below from WALS shows that tonal languages make up over $40 \%$ of the investigated languages and they are far from the minority.

This case study of DH implementation in a linguistic course is a demonstration of our concept of DH accent pitches specifically for undergraduate level English curriculum in the situation of Hong Kong. The Anglo-American publishing industry dominates the international arena of academic publishing and thus textbooks and their examples tend to intellectually incline towards a mono-cultural view. The case we reported above shows that we have derived a DH accent which enables tackling of localised subject matters that meet the needs of local students of English studies in Hong Kong situated in the outer-circle setting. By employing data-driven and digital methods for contrastive linguistic analysis, localised and customised subject matters and linguistic problems highly relevant to postcolonial context of Hong Kong can be explored and presented to students systematically and effectively.

Student projects are often indicative of new and experimental approaches. An interesting example of $\mathrm{DH}$ as a solution comes from a student project combining literary and linguistic studies titled "A study on the diction choice of atmosphere-building in English Gothic 
literature". With the help of corpus tools, such as AntConc, the project investigates five classic English Gothic novels (Carmilla, Dracula, Frankenstein, The Castle of Otranto and The Fall of the House of Usher) and demonstrates some common patterns in the genre. For example, table 1 below lists the words commonly found in the genre:

TABLE 1. Function and choice of diction

\begin{tabular}{ll}
\hline $\begin{array}{l}\text { Function of commonly found } \\
\text { words }\end{array}$ & Examples \\
\hline Specifying time or duration & $\begin{array}{l}\text { night, day, moment, morning, days, half, three, late, years, clock, hour, } \\
\text { September, sun, instant, minutes, morrow, evening }\end{array}$ \\
$\begin{array}{l}\text { Describing indoor } \\
\text { environment }\end{array}$ & room, door, house, window, bed, home, castle, ground, dark \\
$\begin{array}{l}\text { Environment and imageries } \\
\text { life, god, death, dead, light, dark, soul, air, world, earth, sun, water, nature, } \\
\text { sea, cold, wind, wolves } \\
\text { Others }\end{array}$ & heart, fear, blood, white, terrible, red, black, doubt, horror, pale, coffin \\
\hline
\end{tabular}

The student project also includes quantitative data on the number of tokens of these words. While the results are not surprising for readers familiar with gothic novels, the project showcases the strength of digital methods in the accented DH environment. Since some of the concepts and words are not typically in the student's home/local culture. The use of DH is therefore a powerful tool in illustrating what might be considered obvious to speakers in Anglophone cultures. In the context of English studies in Asia, this helps students understand how abstract concepts such as "atmosphere" are built upon atomic and basic entities like words. We therefore argue that such mixed methods are an integral part of "Accented DH". For second language learners of English, reading between the lines or interpreting implicit and subtle cues can be difficult. This student project shows how authors build the atmosphere of the story. The process can be abstract and hard to define, but because of the concrete examples with frequencies, students can therefore identify the cues without resorting to their hunch and intuition.

The other innovation in this project is the use of collocation as a measurement of atmosphere building. Among the top 20 most frequently used collocation types of "sun", one can see 19 tokens of "down", "set" and "setting" and 15 tokens of "up", "high" and "rose". With the frequency data, one can objectively observe and compare the frequencies of the motifs of "sunrise" and "sunset", supplementing the more traditional qualitative "close reading" approach. An important implication here is that the project uses DH techniques and corpus tools to complement qualitative method, rather than replacing it. This demonstrates the strength of mixed-method research: The quantitative corpus tools explore the data set and identify tokens of interest; the qualitative "close reading" approach interprets the general pattern of the text in a more holistic manner. More importantly, the DH methods show an approach with clear steps that can be replicated and extended to other text genres. By analogy, students can apply this method to other genres, such as detective or horror stories or even other text types, and identify the keywords that may appear in these respective genres. This is a clear example of how skills of using observable cues to identify more abstract attributes of a text are complemented by DH methods, and how they can be extended to more generic and transferable skills outside of literary studies.

In short, this case demonstrates how DH techniques benefit English literary studies in an L2 context. With the special setting of English studies in Hong Kong where its curriculum combines the disciplines of literature and linguistics, our proposed accented DH plays the 
crucial role of bridging these two disciplines. DH accent stands for the use of digital tools to help students channel, apply and integrate the methodology, skills and knowledge acquired in linguistic studies in/to their study of literature. At the same time, the formal methods introduced by $\mathrm{DH}$ enhance the metalinguistic awareness of these literature students who, however proficient they are, are L2 learners of English.

\section{CONCLUDING REMARKS}

In this paper, we have argued for the importance of blending digital methods and humanities in a cultural background other than English-dominant ones. With the examples in the context of Hong Kong, in which most students are experienced learners of English and yet the dominant, local culture is Chinese. This situation is representative in various regions in Asia, where instructors have to find a balance between the English language materials and the local culture, so that the materials are both relevant and engaging to students.

We also argue that digital methods and the local accent benefit each other. On one hand, the use of DH provides opportunities to explore outside of the "default" materials that focus on English language or Anglophone cultures. On the other hand, "accented humanities" and its focus on variations can motivate students to take advantage of digital methods for their efficiency, as demonstrated in the inclusion of materials on big data from the World Atlas of Language Structures or the application of corpus methods in studying different Englishes. The more streamlined workflow that comes with technology allows for quick results and feedback that students can see almost instantly. In the time when instructors often have to compete against distraction from social media or mobile games, both within and outside the classroom, technology is useful in channelling students' attention to the class materials with a similar level of instant gratification. In other words, the local accent and digital methods benefit each other by making the materials and the methods interconnected and providing students the autonomy of working on the projects at their own pace.

\section{REFERENCES}

Brandão, Saulo Cunha de Serpa, \& Frota, W. N. (2017). On the path to a methodology for the critique of digital literature. Digital Scholarship in the Humanities, 32(2), 225-233.

Chen, K.-H., \& Hsueh, B.-S. (2014). Exploring Regional Development of Digital Humanities Research: A Case Study for Taiwan. Journal of Data Mining and Digital Humanities, l(1). Retrieved June 30, 2018, from arXiv: $1312.5817 \mathrm{v} 3$.

Cohen, P. (2010). Digital Keys for Unlocking the Humanities' Riches. New York Times, 16.

Cosgrave, M. (2019). Digital humanities methods as a gateway to inter and transdisciplinarity. Global Intellectual History, 1-10.

Dalbello, M. (2011). A Genealogy of Digital Humanities. Journal of Documentation.

Earhart, A. (2018). Digital Humanities Within a Global Context: Creating Borderlands of Localized Expression. Fudan Journal of Humanities and Social Sciences 11(3), 357-369.

Eckert, P. (2008). Variation and the indexical field. Journal of Sociolinguistics 12(4), 453-476.

Fiormonte, D. (2012). Towards a cultural critique of the digital humanities. Historical Social Research/Historische Sozialforschung, 59-76.

Galina, I. (2014). Geographical and linguistic diversity in the digital humanities. Literary and Linguistic Computing, 29(3): 283-306.

Ganapathy, M., Singh, M. K. M., Kaur, S., \& Kit, L. W. (2017). Promoting higher order thinking skills via teaching practices. 3 L: Language, Linguistics, Literature $囚, 23(1)$.

Gold, M. K. (Ed.). (2012). Debates in the Digital Humanities. University of Minnesota Press.

Government of the Hong Kong Special Administrative Region. (2017). 2016 Population By-Census, Hong Kong: Census and Statistics Department. Retrieved from http://www.bycensus2016.gov.hk/en/bc-mt.html

Grice, H. P. (1957). Meaning. The Philosophical Review, 66(3), 377-388. 
Hamat, A., \& Hassan, H. A. (2019). Use of social media for informal language learning by Malaysian university students. 3L: Language, Linguistics, Literature ${ }^{\circledR}, 25(4)$.

Kachru, B. B. (1992). World Englishes: Approaches, issues and resources. Language Teaching, 25(1), 1-14.

Kirschenbaum, M. G. (2016). What is Digital Humanities and What's it Doing in English Departments? Defining Digital Humanities. Routledge, 211-220.

Lin, C., \& Lin, Y. (2019). Grammatical and lexical patterning of make in Asian learner writing: A corpus-based study of ICNALE.3L: Language, Linguistics, Literature ${ }^{\circledR}, 25(3)$

Liu, A. (2013). The meaning of the digital humanities. PMLA, 128(2), 409-423.

Matthews, P. H. (1997). The Concise Oxford Dictionary of Linguistics. Oxford University Press.

Mahony, S. (2018). Cultural Diversity and the Digital Humanities. Fudan Journal of Humanities and Social Sciences 11(3), 371-388.

Perry, M. S. (2018). 21st century skills through film production in tertiary education: A transformative assessment in a literature and media course. 3L: Language, Linguistics, Literature ${ }^{\circledR}, 24(4)$.

Risam, R. and Koh, A. (2013) Postcolonial Digital Humanities. http://dhpoco.org.

Risam, R. (2016) Navigating the Global Digital Humanities: Insights from Black Feminism. Manuscript retrieved July 17, 2018 http://digitalcommons.salemstate.edu/english_facpub/5

Risam, R. (2017). Other worlds, other DHs: Notes towards a DH accent. Digital Scholarship in the Humanities, 32(2), 377-384.

Schreibman, S., Siemens, R., \& Unsworth, J. (Eds.). (2008). A Companion to Digital Humanities. John Wiley \& Sons.

Svensson, P. (2012). The digital humanities as a humanities project. Arts and Humanities in Higher Education, 11(1-2), 42-60. 